\title{
Bacterial sensors synergize to seal T-cell fate
}

The recent discovery of a new $\mathrm{T}$ helper $\left(\mathrm{T}_{\mathrm{H}}\right)$-cell subset that produces interleukin-17 (termed the $\mathrm{T}_{\mathrm{H}} 17$-cell subset) and that is involved in antimicrobial immunity and chronic inflammatory diseases has triggered a flurry of activity aimed at understanding when and how these cells are induced. In mice, transforming growth factor- $\beta$ (TGF $\beta$ ) and interleukin-6 (IL-6) are thought to drive $\mathrm{T}_{\mathrm{H}} 17$-cell differentiation from naive $T$ cells, whereas in humans, studies suggest that $\mathrm{T}_{\mathrm{H}} 17$ cell production is driven by IL-1, IL-6 and/or IL-23. Now, van Beelen et al. show that stimulation of the intracellular bacterial sensor NOD2 (nucleotide-binding oligomerization domain protein 2) programmes dendritic cells (DCs) to promote IL-17 production by human memory T cells.

First, van Beelen et al. showed that human monocyte-derived DCs exposed to various bacterial strains but not viruses were able to induce high levels of IL-17 production in T-cell cultures. Then, by testing a range of known bacterial and viral ligands for Toll-like receptors (TLRs), they established that the most potent component eliciting the IL-17 response was the TLR2 ligand peptidoglycan (PGN), which is present in the cell walls of both Gram-negative and Gram-positive bacteria.

Given that, after internalization, PGN can be metabolized into muramyl dipeptide (MDP), which is a ligand for the intracellular pattern-recognition receptor

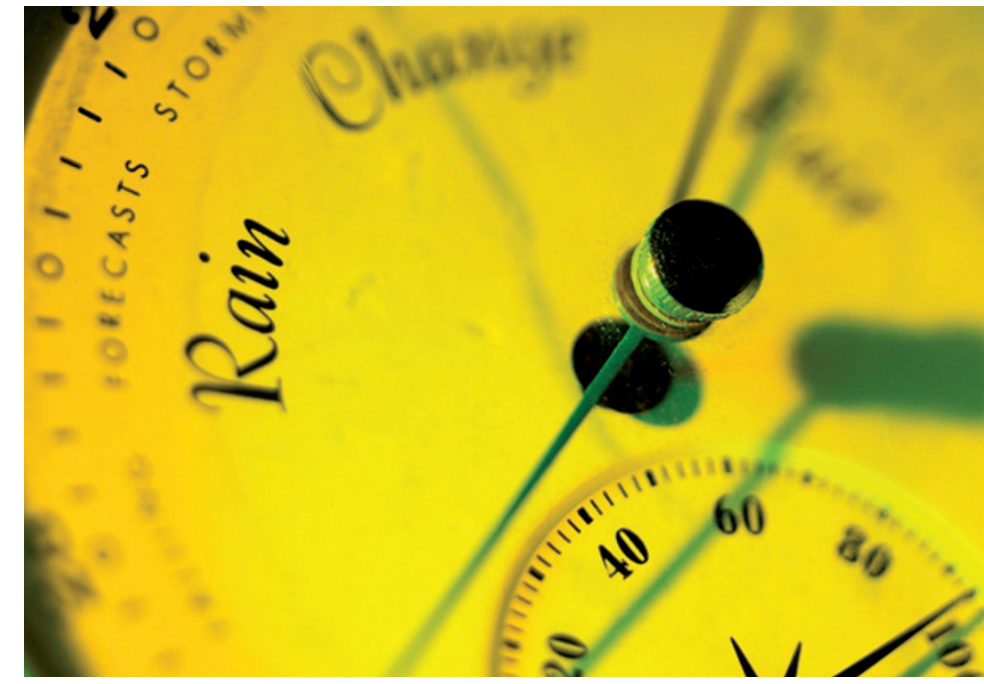

NOD2, the authors next tested whether this pathway was involved in the observed IL-17 response. They showed that when DCs were stimulated with MDP in combination with various TLR ligands, their capacity to specifically promote IL-17 production by memory $\mathrm{T}$ cells was significantly enhanced. This was shown to occur through the induction of IL-23p19, IL-1 $\alpha$ and IL- $1 \beta$ production by the DCs, as previously suggested.

A role for NOD2 in the synergistic effect of MDP was confirmed using monocyte-derived DCs from patients with Crohn's disease carrying a homozygous NOD2 mutation that impairs ligand binding. Indeed, MDP was unable to enhance the IL-17-inducing capacity of TLRprimed DCs from these patients and failed to upregulate IL-23p19 and IL-1 expression.
Surprisingly, contrary to previous studies, the authors noted that the $\mathrm{T}_{\mathrm{H}} 17$ cells induced by IL- 1 and IL-23 did not arise from the differentiation of human naive $\mathrm{T}$ cells, but from the conversion of memory $\mathrm{T}$ cells.

So, NOD2 provides a key link between bacterial infection and the induction of a protective $T_{H} 17$-cell response. How this pathway is involved in the pathogenesis of Crohn's disease needs further investigation, but the recent identification of the IL-23 receptor as a susceptibility gene in this disease is an interesting prospect.

Lucy Bird

ORIGINAL RESEARCH PAPER van Beelen, A. J. et al. Stimulation of the intracellular bacterial sensor NOD2 programs dendritic cells to promote interleukin-17 production in human memory T cells. Immunity 27, 660-669 (2007) FURTHER READING Stetson, D. B. \& Medzhitov, R. Thelper 17 cells get the NOD. Immunity 27 , 546-548 (2007) 